ASTHMA

\title{
Glutathione S transferase deficiency and passive smoking increase childhood asthma
}

\author{
M Kabesch, C Hoefler, D Carr, W Leupold, S K Weiland, E von Mutius
}

Thorax 2004;59:569-573. doi: 10.1136/thx.2003.016667

\begin{abstract}
See end of article for authors' affiliations

....................

Correspondence to: Dr M Kabesch, Children's University Hospital, Ludwig Maximilians University Munich, Lindwurmstrasse 4, D-80337 München, Germany; Michael. Kabesch@med. uni-muenchen.de
\end{abstract}

Received 7 October 2003 Accepted 28 March 2004

\begin{abstract}
Background: It has been suggested that the genetically determined deficiency of glutathione $\mathrm{S}$ transferase (GST) enzymes involved in the detoxification of environmental tobacco smoke (ETS) components may contribute to the development of asthma.

Methods: A large population of German schoolchildren $(n=3054)$ was genotyped for deficiencies of the GST isoforms M1 and T1. The association between GSTM1 and GSTT1 genotypes and asthma as well as atopy was investigated with respect to current and in utero ETS exposure.

Results: In children lacking the GSTM1 allele who were exposed to current ETS the risk for current asthma (OR 5.5, 95\% Cl 1.6 to 18.6) and asthma symptoms such as wheeze ever (OR $2.8,95 \% \mathrm{Cl} 1.3$ to 6.0), current wheezing (OR $4.7,95 \% \mathrm{Cl} 1.8$ to 12.6) and shortness of breath (OR $8.9,95 \% \mathrm{Cl} 2.1$ to 38.4 ) was higher than in GSTM1 positive individuals without ETS exposure. Hints of an interaction between ETS exposure and GSTM1 deficiency were identified. In utero smoke exposure in GSTT1 deficient children was associated with significant decrements in lung function compared with GSTI positive children not exposed to ETS.
\end{abstract}

Conclusions: GSTM1 and GSTT1 deficiency may increase the adverse health effects of in utero and current smoke exposure.
A sthma and allergies have become some of the most frequent chronic diseases in children in western countries. ${ }^{1}$ There is evidence that a genetic predisposition influences the ability of the immune system to interact with the environment. ${ }^{2-4}$ In asthma a genetic predisposition may also alter the capability of the airway to protect itself against inhaled toxic substances from the environment. This may lead to airway inflammation, damage the airway epithelium, and diminish the barrier function of the airway.

Numerous studies have reported an association between environmental tobacco smoke (ETS) exposure and respiratory diseases: Maternal smoking during pregnancy and early childhood is associated with impaired lung growth and diminished lung function ${ }^{5}$ and, in asthmatic children, parental smoking increases symptoms and the frequency of asthma attacks. ${ }^{6}$

ETS consists of a complex mixture of gases and particles including more than 4000 different chemicals. ${ }^{7}$ The metabolism and detoxification of tobacco smoke components are essential mechanisms to minimise the toxic effects of ETS. In humans glutathione $S$ transferases (GSTs) are involved in a broad range of these detoxification processes. ${ }^{8}$ Four cytosolic classes of GSTs have been identified-Alpha (A), Mu (M), Pi $(\mathrm{P})$, and Theta $(\mathrm{T})$-with each of these major classes containing subclasses. For example, five different genes code for $\mathrm{Mu}$ class GSTs (GSTM1-M5). Located mainly in the cytosol, GST enzymes catalyse the conjugation of electrophilic substrates to glutathione, but also play an important role in peroxidase and isomerase activities. ${ }^{8}$ GSTs facilitate responses to oxidative stress reactions, are involved in major detoxification pathways of polycyclic aromatic hydrocarbons, and detoxify benzo[a]pyrene, a carcinogenic found in tobacco smoke. ${ }^{8}{ }^{9}$ It is therefore likely that genetic alterations of GST enzymes change the ability of the airways to deal with toxic substances and influence airway inflammation and lung development. Thus, GST genes have been suggested as candidate genes for asthma. ${ }^{10}$
Genetic studies of the GST system have mainly focused on the genes GSTMI (chromosome lpl3.3) and GSTTl (22q11.2) which code for the enzymes GSTM and GSTT, respectively. ${ }^{10-13}$ Whole gene deletions of GSTMI and GSTT1 resulting in a complete loss of function of the respective enzyme are common in white populations. Previous studies on the effects of genetic alterations in GSTMI and GSTTl have reported associations between these genes and asthma, bronchial hyperresponsiveness, and lung development. ${ }^{10}{ }^{12}$ We genotyped 3054 German children for the genetically determined deficiency of GSTMI and GSTTl and investigated the interaction of both genetic variants with in utero and current ETS exposure in gene $\times$ environment interaction models.

\section{METHODS}

In 1995 and 1996 a cross sectional study was conducted in Munich and Dresden, Germany to assess the prevalence of asthma and allergies in schoolchildren aged 9-11 years as part of the worldwide effort to determine the prevalence of asthma and allergy during childhood. ${ }^{14}$ Parental questionnaires for self-completion including the ISAAC core questions were distributed through the schools to the parents to assess respiratory health and exposure to ETS. Children underwent skin prick testing, pulmonary function testing, and bronchial challenge with hyperosmolar saline (4.5\%). Blood was obtained for serum IgE measurements and DNA extraction. ${ }^{14}$ Total serum IgE was measured in a 50\% random sample of all the children who had given blood in Dresden and all children with blood available in Munich. In this study only children of German origin who had both DNA and $\operatorname{IgE}$ data available were included in the analysis (total $\mathrm{N}=3099$, Munich

Abbreviations: ETS, environmental tobacco smoke; GST, glutathione $S$ transferase; MEF, maximum expiratory flow; MMEF, maximum mid expiratory flow 
$n=1159$, Dresden $n=1940)$. The study methods for all phenotyping procedures have been described in detail elsewhere. $^{214} 15$ To test simultaneously for the presence or absence of GSTMI and GSTTI genes, a modified allele specific PCR assay described previously was used. ${ }^{16}$ Additional information on the applied methods for phenotyping and genotyping is available as supplementary online information on the Thorax website at www.thoraxjnl.com/ supplemental.

\section{Analysis of data}

$\chi^{2}$ statistics and Cochran-Armitage trend tests were used to compare qualitative traits between groups. Total serum IgE levels were summarised descriptively with geometric means and $95 \%$ confidence intervals. For binary outcomes, logistic regression models for gene $\times$ environment interactions were used to estimate the combined effect of either GSTMI or GSTT1 with exposure to smoking in pregnancy and currently, adjusting for the effects of age, sex, city, and family history (asthma, atopic rhinitis, or atopic dermatitis). This analysis used the Botto-Khoury approach which summarises the data in a $2 \times 4$ table, enabling evaluation of the independent and combined roles of genotype and exposure on disease risk. The relative risk estimates for each factor alone and for the combined exposure are comparable because of the use of a common reference group. Additionally, the departure from the multiplicative model of interaction was derived from the table. ${ }^{17}$ Similarly, for continuous outcomes (lung function variables) a two way analysis of covariance (ANCOVA) was carried out to evaluate possible gene $\times$ environment interactions, also adjusting for the possible confounders mentioned above. With several outcomes, two genotypes, and two smoking exposures, the problem of multiple testing needed to be addressed. The Benjamini-Liu method controls the false discovery rate (FDR), a criterion described in Reiner et $a l^{18}$ within a predefined family of tests. We used the Benjamini-Liu multiple test correction with families for asthma, wheeze, treatment, and lung function for each smoking exposure and each genotype; within each family the FDR was controlled to either $1 \%$ or $5 \%$ (fig $1 \mathrm{~A}$ and B).

All calculations were carried out using the SAS software package (version 8.2).

\section{RESULTS}

The study population successfully genotyped for this analysis consisted of 3054 subjects who were all of German nationality and origin. Of these, $1140(37.3 \%)$ were from Munich, western Germany, and 1914 (62.7\%) were from Dresden, eastern Germany. With respect to the prevalence of atopic diseases and the distribution of age, sex, and socioeconomic markers, the population from both areas did not differ from the subgroup which was not genotyped (data not shown). However, ETS exposure in utero as well as in the current household of the child was significantly more prevalent in Munich than in Dresden (in utero: 14.2\% $v$ 4.4\%; current ETS: $5.3 \%$ v $2.0 \%$ )

The frequency of the GSTMI null genotype was $51.6 \%$ (Munich 51.9\%, Dresden 51.4\%) and the frequency of the GSTT1 null genotype was $17.3 \%$ (Munich 16.3\%, Dresden $17.8 \%$ ). Double null genotypes (concomitant absence of both GSTMI and GSTTl alleles) occurred in 8.8\% of the genotyped samples (Munich 7.8\%, Dresden 9.4\%). No statistically significant difference in allele frequency between the study populations from Munich and Dresden was detected. The allele distribution was within the range of previously reported allele frequency of GSTMI and GSTT1 null alleles in other white populations. ${ }^{12}$ Furthermore, the distribution of genotypes in the population did not deviate from the expected Hardy-Weinberg equilibrium.
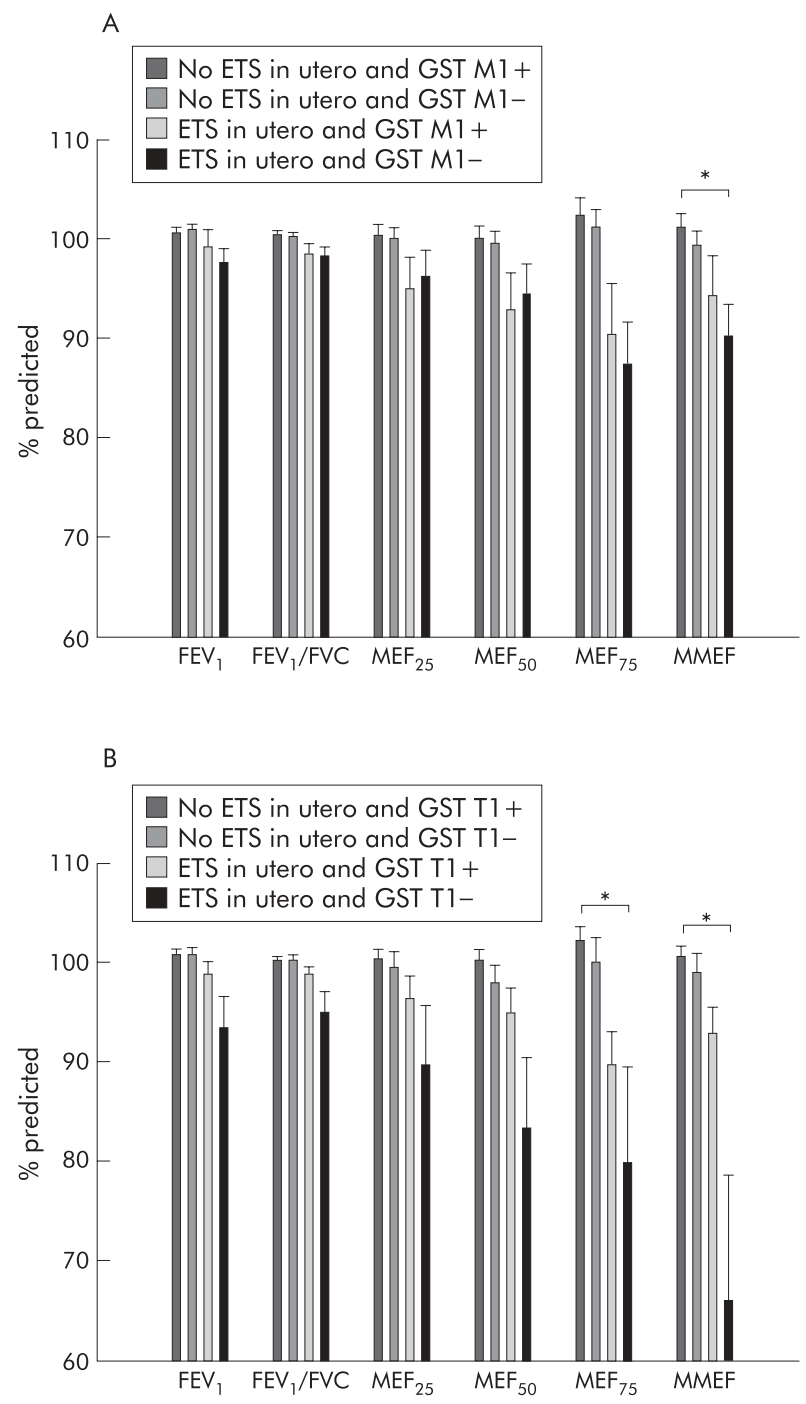

Figure 1 Combined effects on pulmonary function (\% predicted) of (A) GSTMI and (B) GSTT1 genotypes and exposure in utero to environmental tobacco smoke (ETS). *False discovery rate (FDR) $\leqslant 5 \%$; statistically significant after Benjamini-Liu multiple testing correction.

We did not detect a major effect of GSTMI or GSTTl null genotypes per se on the prevalence of asthma, asthma symptoms, wheezing, or other atopic diseases in our unstratified population sample of German children (table El, online supplement). Furthermore, no effect of a double null GST genotype on the prevalence of atopic diseases was observed (data not shown). For the development of childhood asthma a gene $\times$ gene interaction model based on GSTMI and GSTTl was rejected (data not shown).

When a combined analysis of smoke exposure and GSTMI genotypes was performed, a significantly increased risk of developing asthma, asthma symptoms, and wheezing was apparent in children who had a GSTMl or GSTTl null genotype and were also exposed to current ETS (tables 1 and $2)$. In children with a GSTMl null genotype exposed to current ETS the increased risk was statistically significant for all wheeze outcomes as well as for current asthma. For children with a GSTT1 null genotype exposed to current ETS, odds ratios (ORs) similar to those found in GSTMl were observed. However, as the gene frequency was lower, confidence intervals were wider and the trends were not always statistically significant. In tables 1 and 2 , expected 
Table 1 Botto-Khoury $2 \times 4$ table for analysis of gene $\times$ environment interactions for GSTM1 genotypes versus current ETS exposure ( $\geqslant 20$ cigarettes)

\begin{tabular}{|c|c|c|c|c|c|}
\hline Outcomes & (1) GSTM1+ and ETS- & (2) GSTMI+ and ETS+ & (3) GSTM1 - and ETS- & (4) GSTMI - and ETS+ & $\begin{array}{l}\text { (5) Expected OR } \mathrm{O} / \text { departure } \\
\text { from expected OR ( } ¥ \ddagger)\end{array}$ \\
\hline \multirow[t]{2}{*}{ Current asthma } & $3.9(29 / 735)$ & $6.1(2 / 33)$ & $5.1(39 / 758)$ & $8.9(4 / 45)$ & \\
\hline & & $2.94(0.61$ to 14.05$)$ & $1.40(0.85$ to 2.30$)$ & $5.48(1.62 \text { to } 18.55)^{*}$ & $4.10 / 1.34(p=0.77)$ \\
\hline \multirow[t]{2}{*}{ Wheeze ever } & $19.6(142 / 725)$ & $24.2(8 / 33)$ & $23.0(172 / 748)$ & $31.1(14 / 45)$ & \\
\hline & & $1.81(0.75$ to 4.41$)$ & $1.27(0.98$ to 1.64$)$ & $2.81(1.31 \text { to } 6.04)^{*}$ & $2.30 / 1.22(p=0.71)$ \\
\hline \multirow{2}{*}{ Current wheeze } & $6.5(47 / 726)$ & $9.1(3 / 33)$ & $8.9(67 / 749)$ & $17.8(8 / 45)$ & \\
\hline & & $2.03(0.55$ to 7.54$)$ & $1.50(1.01 \text { to } 2.22)^{*}$ & $4.74(1.79 \text { to } 12.57)^{*}$ & $3.04 / 1.56(p=0.56)$ \\
\hline \multirow{2}{*}{$\begin{array}{l}\text { Current cough } \\
\text { without cold }\end{array}$} & $15.5(113 / 727)$ & $12.1(4 / 33)$ & $15.5(117 / 753)$ & $26.1(12 / 46)$ & \\
\hline & 1.0 & 1.04 (0.34 to 3.18 ) & 1.03 (0.78 to 1.37 ) & 2.76 (1.23 to 6.17$)^{*}$ & $1.07 / 2.58(p=0.15)$ \\
\hline \multicolumn{6}{|c|}{$\begin{array}{l}\text { In each cell the prevalence (\%) of each outcome is shown with numbers of cases and total in parentheses. Adjusted odds ratio (OR) with } 95 \% \text { confidence intervals, } \\
\text { adjusted for age, sex, city, family history of asthma, hay fever, eczema and ETS exposure, are compared with the reference cell (second line of each cell). Column } \\
1 \text { shows the reference group without smoke exposure and without a genetic defect in the respective GST enzyme; column } 2 \text { shows the effect of GST deficiency } \\
\text { alone; column } 3 \text { shows the isolated effect of ETS exposure in children with the intact GST gene, respectively; column } 4 \text { shows the values for the combined effect of } \\
\text { the respective GST deficiency and concomitant ETS exposure; column } 5 \text { shows the expected OR under the assumption of a multiplicative effect, the departure from } \\
\text { the effect, and the statistical significance of the departure from the multiplicative model. } \\
\text { "p value }<0.05 \text { compared with reference group. } \\
\text { †Departure from expected OR }=\text { OR in column } 4 \text { divided by the expected OR. } \\
\text { tp value from the test for interaction from logistic regression model. } \\
\text { \$Expected OR is the product of the ORs in columns } 2 \text { and } 3 \text {. }\end{array}$} \\
\hline
\end{tabular}

ORs for the combined effects of ETS exposure and GSTMI/ GSTTI deficiency are shown as well as departures from the expected OR for multiplicative interaction (column 5). As shown in table 1 , the effect of the GSTMI null genotype without ETS exposure was only marginal, while exposure to ETS alone increased the risk for current asthma and asthma symptoms regardless of the GSTMI genotype. A combined statistically significant effect of the GSTMI null genotype and ETS exposure was observed compared with children with no ETS exposure and no GSTMl deficiency (column 4), with several variables showing a positive departure from the expected OR for multiplicative interaction. However, this departure did not reach statistical significance, thus only hinting at a possible interaction (column 5).

Exposure to in utero ETS combined with a GSTMl null genotype showed a trend for an increased risk without reaching statistical significance, while the combination of a GSTT1 null genotype and in utero ETS showed no obvious pattern (tables E2 and E3, online supplement). In the analysis of current smoke exposure and GST genotypes, asthma symptoms increased with the amount of current smoke exposure with the strongest effect being observed in children exposed to more than 20 cigarettes smoked in the household (data not shown).
In a further analysis the combined effect of current or in utero ETS exposure and GST genotypes on lung function measurements was studied (fig $1 \mathrm{~A}$ and B). In utero ETS exposure combined with the GSTT1 null genotype showed the strongest effect on lung function measurements (fig lA). All other lung function measurements showed small to very large decreases in the GSTTl negative subgroup exposed to ETS in utero. After correction for multiple testing, a statistically significant interaction between ETS exposure and GSTTl deficiency was observed for the $\mathrm{MEF}_{75}$ and MMEF measurements. The GSTMI null genotype and in utero ETS exposure showed a weaker combined effect on lung function (fig 1B), statistically significant only in its effect on MMEF. Current ETS exposure combined with GST deficiency did not decrease lung function measurements in our study population (data not shown).

\section{DISCUSSION}

This study has genotyped the largest population to date for polymorphisms in GSTTl and GSTM1. In contrast to previous studies, ${ }^{12}$ all 3054 eligible study subjects were white and of German nationality and origin. Population stratification is therefore very unlikely to have influenced the findings. The population was carefully phenotyped for asthma and allergic

Table 2 Botto-Khoury $2 \times 4$ table for analysis of gene $\times$ environment interactions for GSTT1 genotypes versus current ETS exposure ( $\geqslant 20$ cigarettes)

\begin{tabular}{|c|c|c|c|c|c|}
\hline Outcomes & (1) GSTI 1+ and ETS- & (2) GSTTI+ and ETS+ & (3) GSTT1 - and ETS- & (4) GSTT I - and ETS+ & $\begin{array}{l}\text { (5) Expected OR\&/departure } \\
\text { from expected OR ( } ¥ \ddagger)\end{array}$ \\
\hline \multirow[t]{2}{*}{ Current asthma } & $4.8(59 / 1238)$ & $7.5(5 / 67)$ & $3.5(9 / 255)$ & $9.1(1 / 11)$ & \\
\hline & 1.0 & $3.25(1.14 \text { to } 9.27)^{*}$ & $0.73(0.35$ to 1.50$)$ & $4.10(0.43$ to 39.04$)$ & $2.37 / 1.73(p=0.67)$ \\
\hline \multirow{2}{*}{ Wheeze ever } & $21.8(266 / 1222)$ & $25.4(17 / 67)$ & $19.1(48 / 251)$ & $45.5(5 / 11)$ & \\
\hline & 1.0 & $1.77(0.92$ to 3.42$)$ & $0.84(0.60$ to 1.19$)$ & $4.37(1.17 \text { to } 16.39)^{*}$ & $1.49 / 2.93(p=0.13)$ \\
\hline \multirow[t]{2}{*}{ Current wheeze } & $7.9(96 / 1222)$ & $13.4(9 / 67)$ & $7.1(18 / 253)$ & $18.2(2 / 11)$ & \\
\hline & 1.0 & $2.61(1.09 \text { to } 6.21)^{*}$ & $0.90(0.53$ to 1.52$)$ & $3.30(0.61$ to 17.91$)$ & $2.34 / 1.41(p=0.71)$ \\
\hline \multirow{2}{*}{$\begin{array}{l}\text { Current cough } \\
\text { without cold }\end{array}$} & $15.4(189 / 1228)$ & $16.2(11 / 68)$ & $16.3(41 / 252)$ & $45.5(5 / 11)$ & \\
\hline & 1.0 & $1.50(0.71$ to 3.20$)$ & $1.08(0.74$ to 1.56$)$ & $6.72(1.77 \text { to } 25.52)^{*}$ & $1.62 / 4.15(p=0.05)$ \\
\hline
\end{tabular}

In each cell the prevalence (\%) of each outcome is shown with numbers of cases and total in parentheses. Adjusted odds ratio (OR) with 95\% confidence intervals, adjusted for age, sex, city, family history of asthma, hay fever, eczema and ETS exposure, are compared with the reference cell (second line of each cell). Column 1 shows the reference group without smoke exposure and without a genetic defect in the respective GST enzyme; column 2 shows the effect of GST deficiency alone; column 3 shows the isolated effect of ETS exposure in children with the intact GST gene, respectively; column 4 shows the values for the combined effect of the respective GST deficiency and concomitant ETS exposure; column 5 shows the expected OR under the assumption of a multiplicative effect, the departure from the effect, and the statistical significance of the departure from the multiplicative model.

*p value $<0.05$ compared with the reference group.

†Departure from expected OR = OR in column 4 divided by the expected OR.

$\ddagger p$ value from the test for interaction from logistic regression model.

$\S$ Expected OR is the product of the ORs in columns 2 and 3. 
traits according to the international standards of the ISAAC study protocols, and the validity of questionnaire derived phenotypes has been shown previously. ${ }^{14}$ The exposure of children to ETS in the home was assessed by selfadministered parental questionnaires shown to be a valid tool for measuring ETS exposure. ${ }^{19} 20$

When ETS exposure was not included in the analysis, neither GSTMI nor GSTTl deficiency had an effect on the development of asthma in this population (table El, online supplement). These findings indicate that the environmental exposure to toxic substances is necessary to unravel the effect of the genetically determined deficiencies in GST dependent detoxification processes. In GSTMI deficient children exposed to ETS, odds ratios for current asthma, wheezing phenotypes, and current cough were significantly increased while, for GSTT1 deficient children exposed to ETS, only wheeze ever and current cough reached statistical significance. When interaction models were applied as shown in the Botto-Khoury tables, an overall trend for a positive effect was found, mostly over and above the expected multiplicative interaction between GSTMI and GSTTl deficiency and ETS exposure. Even though the observed combined ORs deviated consistently from expected multiplicative models, the deviation above the expected multiplicative OR did not reach statistical significance. This may largely be the result of the relatively small number of subjects in the high risk groups, even though more than 3000 subjects were initially included and genotyped in this project. Power calculations indicate that, based on our baseline disease prevalence rates, between 2.7 and 48 times more individuals would have been necessary to reach statistical significance for all the observed interaction effects. However, the statistically suggested interaction between ETS exposure and GSTMI deficiency constantly shown in our data adds to the plausibility of a biological interaction between GST enzymes and components of ETS in the detoxification process. Experimental data support these epidemiological observations: In the lung tissue of GSTMI deficient individuals higher levels of aromatic DNA adducts have been found, ${ }^{21}$ and cytogenetic damage to lung cells caused by smoke exposure increases with GSTMI deficiency. ${ }^{22}$ This indicates increased damage to DNA and the destruction of tissue due to diminished GSTMl function. Also, GSTTl negative individuals have been found to have significantly higher levels of DNA damage than GSTT1 positive individuals in experimental in vitro settings. ${ }^{23}$ Furthermore, recent data indicate that GSTMI may modify the adjuvant effect of diesel exhaust particles on allergic inflammation. ${ }^{24}$

Our findings suggest that the strongest effect on the development of asthma and asthma symptoms occurs in GSTMI and GSTTI deficient individuals currently exposed to ETS. However, a small non-significant effect of in utero smoke exposure in GSTMl deficient children on the occurrence of asthma and asthma symptoms was also observed in this population (tables E2 and E3, online supplement). In contrast to our findings, Gilliland and coworkers reported a major effect of in utero ETS exposure on asthma and asthma symptoms in GSTMl negative individuals in their population of children from the US, whereas current ETS exposure showed only a non-significant increase in asthma and wheeze. ${ }^{12}$ The combination of these findings indicates that GST negative individuals are at a higher risk of developing asthma and asthma symptoms which could be the result of in utero as well as current ETS exposure. As both exposures are interconnected, our analyses for the effects of in utero ETS exposure were adjusted for current ETS exposure and vice versa. Both types of ETS exposure showed independent effects on respiratory health in GSTMI negative individuals in our analysis, but were only significant for current passive smoking where a trend for a dose dependent effect was observed (data not shown). Unfortunately, children heavily exposed to ETS in utero could not be identified in our study as the extent of active and passive smoking of mothers during pregnancy was not assessed.

Evidence exists that smoking during pregnancy affects the growth of the fetus and the maturation of the fetal lung, resulting in an impairment of lung function in the newborn. ${ }^{25}$ Furthermore, previous studies have suggested that GSTMI deficient children may have impaired lung function growth. ${ }^{11}$ Our results suggest, however, that pulmonary function is only affected in exposed subjects with a GSTTl deficiency. Interestingly, small airways were affected most while forced expiratory volume in l second measurements remained unaffected. As shown previously, early deficits in small airway function may predispose children to transient wheezing later in life. ${ }^{5}$ Our data allowed us to define different wheezing phenotypes retrospectively. Only wheezing persisting from infancy to school age was associated with GSTTl deficiency and ETS exposure (data not shown), which suggests that small airway size may contribute to the development of asthma. Since prevalence is determined by incidence and duration of an illness, we cannot discriminate whether the observed effects relate more to the severity or to the incidence of asthma. While ETS exposure is a serious health hazard for children in the general population, some are even more susceptible to developing asthma and more severe asthma symptoms when exposed to passive smoking. GST deficiency and other genetic alterations in detoxification pathways not yet studied may add to the adverse health effects caused by passive and active tobacco smoking which should be further investigated in large population samples.

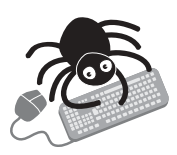

Additional information is available as supplementary online information on the Thorax website at www.thoraxjnl.com/supplemental.

\section{Authors' affiliations \\ M Kabesch, C Hoefler, D Carr, E von Mutius, University Children's Hospital Munich, Germany \\ W Leupold, University Children's Hospital Dresden, Germany S K Weiland, Institute of Epidemiology, University of Ulm, Germany}

This study was funded by the German Ministry of Education and Research as part of the German national genome research network, grant GS 010122 . MK was also funded by the carrier development grant R186 from the University of Munich.

\section{REFERENCES}

1 International Study of Asthma and Allergies in Childhood (ISAAC) Steering Committee. Worldwide variation in prevalence of symptoms of asthma, allergic rhinoconjunctivitis, and atopic eczema: ISAAC. Lancet 1998:351:1225-32.

2 Kabesch M, Peters W, Carr D, et al. Association between polymorphisms in caspase recruitment domain containing protein 15 and allergy in two German populations. J Allergy Clin Immunol 2003;11 1:813-7.

3 Baldini M, Lohman IC, Halonen M, et al. A polymorphism in the $5^{\prime}$ flanking region of the $C D 14$ gene is associated with circulating soluble CDI 4 levels and with total serum immunoglobulin E. Am J Respir Cell Mol Biol 1999;20:976-83.

4 Lazarus R, Vercelli D, Palmer $\sqcup$, et al. Single nucleotide polymorphisms in innate immunity genes: abundant variation and potential role in complex human disease. Immunol Rev 2002;190:9-25.

5 Cook DG, Strachan DP, Carey IM. Health effects of passive smoking. 9. Parental smoking and spirometric indices in children. Thorax 1998;53:884-93.

6 Cook DG, Strachan DP. Health effects of passive smoking. 3. Parental smoking and prevalence of respiratory symptoms and asthma in school age children. Thorax 1997;52:1081-94.

7 Lofroth G. Environmental tobacco smoke: overview of chemical composition and genotoxic components. Mutat Res 1989;222:73-80. 
8 Sheehan D, Meade G, Foley VM, et al. Structure, function and evolution of glutathione transferases: implications for classification of non-mammalian members of an ancient enzyme superfamily. Biochem J 2001;360:1-16.

9 Alexandrov K, Cascorbi I, Rojas M, et al. CYPIA1 and GSTM1 genotypes affect benzo[a]pyrene DNA adducts in smokers' lung: comparison with aromatic/hydrophobic adduct formation. Carcinogenesis 2002;23:1969-77.

10 Ivaschenko TE, Sideleva OG, Baranov VS. Glutathione- S-transferase micro and theta gene polymorphisms as new risk factors of atopic bronchial asthma. $\mathrm{J}$ Mol Med 2002;80:39-43.

11 Gilliland FD, Gauderman WJ, Vora H, et al. Effects of glutathione-Stransferase $\mathrm{M} 1, \mathrm{Tl}$, and $\mathrm{Pl}$ on childhood lung function growth. Am J Respir Crit Care Med 2002;166:710-6.

12 Gilliland FD, Li YF, Dubeau L, et al. Effects of glutathione S-transferase MI, maternal smoking during pregnancy, and environmental tobacco smoke on asthma and wheezing in children. Am J Respir Crit Care Med 2002; 166:457-63.

13 Menegon A, Board PG, Blackburn AC, et al. Parkinson's disease, pesticides, and glutathione transferase polymorphisms. Lancet 1998;352:1344-6.

14 Weiland SK, von Mutius E, Hirsch T, et al. Prevalence of respiratory and atopic disorders among children in the East and West of Germany five years after unification. Eur Respir J 1999;14:862-70

15 von Ehrenstein OS, von Mutius E, Maier E, et al. Lung function of school children with low levels of alphal-antitrypsin and tobacco smoke exposure. Eur Respir J 2002;19:1099-106.

16 Chen CL, Liu Q, Relling MV. Simultaneous characterization of glutathione Stransferase $\mathrm{Ml}$ and $\mathrm{Tl}$ polymorphisms by polymerase chain reaction in American whites and blacks. Pharmacogenetics 1996;6:187-91.
17 Botto LD, Khoury MJ. Commentary: facing the challenge of gene-environment interaction: the two-by-four table and beyond. Am J Epidemiol 2001;153:1016-20.

18 Reiner A, Yekutieli D, Benjamini Y. Identifying differentially expressed genes using false discovery rate controlling procedures. Bioinformatics 2003; 19:368-75.

19 Oddoze C, Dubus JC, Badier M, et al. Urinary cotinine and exposure to parental smoking in a population of children with asthma. Clin Chem 1999;45:505-9.

20 Cook DG, Whincup PH, Papacosta O, et al. Relation of passive smoking as assessed by salivary cotinine concentration and questionnaire to spirometric indices in children. Thorax 1993;48:14-20.

21 Ryberg D, Skaug V, Hewer A, et al. Genotypes of glutathione transferase MI and $\mathrm{Pl}$ and their significance for lung DNA adduct levels and cancer risk. Carcinogenesis 1997; 18:1285-9.

22 van Poppel G, de Vogel N, van Balderen PJ, et al. Increased cytogenetic damage in smokers deficient in glutathione S-transferase isozyme mu. Carcinogenesis 1992;13:303-5.

23 Ollikainen T, Hirvonen A, Norppa H. Influence of GSTTl genotype on sister chromatid exchange induction by styrene-7, 8-oxide in cultured human lymphocytes. Environ Mol Mutagen 1998;31:311-5.

24 Gilliland FD, Li YF, Saxon A, et al. Effect of glutathione-S-transferase M1 and P1 genotypes on xenobiotic enhancement of allergic responses: randomised, placebo-controlled crossover study. Lancet 2004;363:119-25.

25 Cook DG, Strachan DP. Health effects of passive smoking. 10: Summary of effects of parental smoking on the respiratory health of children and implications for research. Thorax 1999;54:357-66.

\section{LUNG ALERT}

Improved survival with postoperative uracil-tegafur in stage 1 adenocarcinoma of the lung

$\Delta$ Kato $\mathrm{H}$, Ichinose $\mathrm{Y}$, Ohta $\mathrm{M}$, et al. A randomized trial of adjuvant chemotherapy with uracil-tegafur for adenocarcinoma of the lung. N Engl J Med 2004;350:1713-21

$\mathrm{T}$

egafur is a pro-drug that is converted to fluorouracil, and the serum concentration of fluorouracil is enhanced by uracil. While fluorouracil itself has no apparent effect on

survival in non-small cell lung cancer, Japanese studies of uracil-tegafur have reported a mortality benefit when used as an adjuvant therapy. In subgroup analysis this benefit was confined to adenocarcinoma.

The present study randomised patients to receive oral uracil-tegafur $\left(250 \mathrm{mg} / \mathrm{m}^{2} /\right.$ day, $n=491)$ or no treatment $(n=488)$ for 2 years following resection of stage 1 (TIMON0 or T2MON0) adenocarcinoma of the lung. Despite being an open label study, it was well planned and adequately powered. Median follow up was 73 months. Overall there was a statistically significant survival advantage favouring treatment $(\mathrm{p}=0.04)$ with a 5 year survival of $88 \%$ (CI 85 to 91 ) compared with $85 \%$ (CI 82 to 89 ). On subgroup analysis this difference was only apparent in those patients with T2 disease: 5 year survival with treatment was $85 \%$ (CI 79 to 91 ) compared with $74 \%$ without (CI 66 to 81 ). There were few serious drug related adverse events but only $61 \%$ of patients completed the 2 year treatment period.

This study shows a survival advantage for postoperative adjuvant oral uracil-tegafur in stage 1 (T2) adenocarcinoma of the lung.

M H Lawson

Senior House Officer, St Bartholomew's Hospital, London, UK mhlawson@tregarvonroad.fsnet.co.uk 